\title{
Optimal sampling plan for clean development mechanism energy efficiency lighting projects $^{\text {th }}$
}

\author{
Xianming Ye*, Xiaohua Xia, Jiangfeng Zhang \\ Department of Electrical, Electronic and Computer Engineering, University of Pretoria, Pretoria, 0002, South Africa
}

\begin{abstract}
Clean development mechanism (CDM) project developers are always interested in achieving required measurement accuracies with the least metering cost. In this paper, a metering cost minimisation model is proposed for the sampling plan of a specific CDM energy efficiency lighting project. The problem arises from the particular CDM sampling requirement of $90 \%$ confidence and $10 \%$ precision for the small-scale CDM energy efficiency projects, which is known as the 90/10 criterion. The 90/10 criterion can be met through solving the metering cost minimisation problem. All the lights in the project are classified into different groups according to uncertainties of the lighting energy consumption, which are characterised by their statistical coefficient of variance (CV). Samples from each group are randomly selected to install power meters. These meters include less expensive ones with less functionality and more expensive ones with greater functionality. The metering cost minimisation model will minimise the total metering cost through the determination of the optimal sample size at each group. The 90/10 criterion is formulated as constraints to the metering cost objective. The optimal solution to the minimisation problem will therefore minimise the metering cost whilst meeting the $90 / 10$ criterion, and this is verified by a case study. Relationships between the optimal metering cost and the population sizes of the groups, CV values and the meter equipment cost are further explored in three simulations. The metering cost minimisation model proposed for lighting systems is applicable to other CDM projects as well.
\end{abstract}

Keywords: CDM, sample size determination, energy efficiency, lighting

\section{Nomenclature}

$\bar{E}$

$\bar{x}_{i}$

$\lambda_{0}$

$\mu_{i}$

$\sigma_{i}$

$a_{i}$

$b_{i}$

$c_{i}$

$C V_{i}$

$E_{B}$

$E_{i}$

$i$

$k$ the weighted average daily energy consumption per lamp in the project

the sample mean in the $i$-th group

the search starting point to solve the optimisation model

the true mean value in the $i$-th group

the true standard deviation in the $i$-th group, $\sigma_{i}=\bar{x}_{i} C V_{i}$

the individual meter device cost in the $i$-th group the installation cost per meter in the $i$-th group the monthly maintenance cost per meter in the $i$-th group

the estimated coefficient of variance in the $i$-th group

the daily energy consumption baseline (in kWh)

the daily energy consumption per lamp in the $i$-th group

the counter of the subgroups of a project the number of months during the monitoring period

\footnotetext{
A preliminary version of this paper was presented in the International Conference on Applied Energy, July 5-8, 2012, Suzhou, China.

${ }^{*}$ Corresponding author. Tel.: +27 (0)12 420 4353; fax: +27 (0)12 362 5000; Email address: xianming.ye@up.ac.za (Xianming Ye).
}

$M_{i}$

$N$

$N_{i}$

$O_{i}$

$p$

$P_{i}$

$p_{i}$

$z$

$z_{i}$

A

$\mathrm{AC}$

AMS

ASHRAE

CDM

CER

CFL

$\mathrm{CV}$

GHG

HDL

ICL

IPMVP the cost of individual metering system over the crediting period, $M_{i}=a_{i}+b_{i}+k c_{i}$

the lighting population

the number of devices in the $i$-th group

the average daily utilisation hours of devices in the $i$-th group

the required relative precision for the project

the power of devices in the $i$-th group

the precision level in the $i$-th group

the abscissas of the normal distribution curve that cut off an area at the tails to give desired confidence level

the $z$ value in the $i$-th group

ampere

alternating current

approved methodology for small-scale

American society of heating, refrigerating and air-conditioning engineers

clean development mechanism

certified emission reduction

compact florescent lamp

coefficient of variance

greenhouse gas

halogen downlighter

incandescent lamp

international performance measurement and verification protocol 


$\begin{array}{ll}\text { kB } & \text { kilobyte } \\ \text { kWh } & \text { kilowatt-hour } \\ \text { mA } & \text { milliampere } \\ \text { MB } & \text { megabyte } \\ \text { R } & \text { the South African currency Rand } \\ \text { S } & \text { second } \\ \text { SSC } & \text { small-scale } \\ \text { SSD } & \text { sample size determination } \\ \text { TWh } & \text { terawatt-hour } \\ \text { UNFCCC } & \text { united nations framework convention on cli- } \\ & \text { mate change } \\ \text { USD } & \text { the United States dollar } \\ \text { V } & \text { voltage } \\ \text { W } & \text { watt }\end{array}$

\section{Introduction}

CDM projects are designed to assist the reduction of GHG emissions in developing countries by allowing them to earn CER credits which can be traded and used by developed countries [1]. In order to mitigate the climate change and avoid global warming, different types of CDM energy efficiency projects are registered under the UNFCCC, such as the energy efficiency lighting, heat pump and chiller projects [2]. Note that lighting consumes more than 2000 TWh of electricity globally, which corresponds to about 1800 million metric tons of GHG emission per year [3]. Therefore, lighting harbors a great potential for energy savings and GHG emission reductions. According to [4], the energy efficiency lighting projects can be implemented by either reducing input wattages or reducing the utilisation hours of the lights, and these measures are widely taken in residential, commercial and industrial sectors around the world ([5], [6] and [7]).

It is important to quantify CER credits with the expected accuracy in a cost-effective way so that the profits of CDM project developers can be maximised. As presented in both [8] and [9], the deemed savings methodology is adopted in order to alleviate the metering cost and monitoring needs. However, also as indicated in [8], the deemed savings methodology generates less CERs due to very conservative assumptions on the key parameters to determine the baseline and project energy consumption. Apparently, the CERs generated by the deemed savings methodology are only conservative but not accurate. According to the CDM general guidelines [10] and the AMS CDM projects, AMS-II.C. [11], the CER credits are calculated by the corresponding energy consumption reduction multiplied the emission factors. The baseline and project energy consumption need to be quantified by the monitored and sampled key parameters based on the sampling methodologies [12] and [13]. The sampling methodologies [12] and [13] are further required to satisfy $90 \%$ confidence and $10 \%$ precision - the so-called $90 / 10$ criterion $^{1}$ for the SSC energy efficiency CDM projects. For the $\mathrm{CDM}$ accuracy requirement, precision is an assessment of the

\footnotetext{
${ }^{1}$ Following the notation of the $90 / 10$ criterion, $x / y$ denotes $x \%$ confidence and $y \%$ precision in this study.
}

error margin of the final estimate and confidence is the likelihood that the sampling resulted in an estimate within a certain range of the true values.

The purpose of this paper is to maximise the profits of project developers by minimising metering cost while at the same time meet the $90 / 10$ criterion. An obvious observation is that the metering cost is lower whenever less number of samples is metered. For this reason, many different kinds of SSD methodologies have been proposed in literature. These SSD methodologies can be classified into two broad groups, the frequentist methods and the Bayesian methods [14]. The frequentist methods have been applied to determine sample size for the evaluation of the reliability performance of the United States fleet [15] and the control of both size and power in clinic trials [16]. For the Bayesian methods, [17] summaries the theory and practice of Bayesian statistics while [18] describes a Bayesian approach in choosing the sample size by optimising utility functions.

All the aforementioned SSD methodologies are mainly decided by the required confidence and precision level. According to [19], the SSD for the CDM projects is also influenced by different choices of the sampling approaches such as the simple random sampling, stratified random sampling, systematic sampling, cluster sampling and multi-stage sampling, etc. Based on the available information on the UNFCCC's website ${ }^{2}$, more than 20 energy efficiency lighting projects have been registered under SSC CDM projects. All these registered CDM lighting projects refer to the sampling guidelines [12] and [13] to design the monitoring and sampling plans. However, these projects usually apply the simple random sampling approach to determine the sample sizes since the entire lighting population is assumed to be homogeneous and each lights has the same probability of being selected ([20] and [21]).

In this paper, the metering cost minimisation under the situation where the lighting population under study are not homogeneous has been considered. The total population, i.e., all the lights in the project, is divided into different groups according to their energy consumption uncertainties. Therefore, the stratified random sampling approach is applied given that the characteristics of the population elements are more similar within groups than across groups [19]. With the selected stratified random sampling approach, the idea to reduce the overall metering cost whist maintaining the 90/10 criterion is explained as follows. The sampling guidelines [12] and [13] require that the sampling target for the project population must satisfy the 90/10 criterion. However, it does not mean that the accuracy criterion for each lighting group must achieve 90/10. It is possible to let the group with lower uncertainty to have a very good confidence/precision such as $95 / 5$, and let the group with higher uncertainty to have a relative poor confidence/precision such as $85 / 15$, so that the overall project population satisfies the $90 / 10$ requirement. The proposed metering cost minimisation model follows the above idea to determine the optimal confidence/precision levels such that the metering cost can be minimised while the overall $90 / 10$ criterion is still maintained.

\footnotetext{
${ }^{2}$ http://cdm.unfccc.int/methodologies/index.html
} 
This model can be easily applied to various lighting projects with more than two uncertainty groups.

The paper is organised as follows. In Section 2, background information of the CDM lighting project is provided with a total project cost analysis. The lighting baseline methodology, lighting classification, the monitoring and sampling plan for this project are then discussed. Subsequently, a brief uncertainty analysis is given and some essential assumptions are made in order to build the metering cost minimisation model. In Section 3, the solutions to the optimisation problem are provided and analysed. Section 4 provides some further investigations of the metering cost optimisation model. The conclusion comes at the last section.

\section{Formulation of the metering cost minimisation model}

\subsection{Backgrounds of the CDM lighting project}

A lighting retrofit project that aims to reduce the lighting load in 44 government hospitals in South Africa is in the process of being registered as a CDM energy efficiency lighting project. In order to accelerate the registration process, the project developers decide to register the project as a SSC CDM project. Approximately 404296 CFLs will be installed to replace less energy efficient HDLs and ICLs that are currently in use. The $14 \mathrm{~W}$ and $20 \mathrm{~W}$ CFLs will be installed in exchange of equal number of normal luminous flux $60 \mathrm{~W}$ ICLs and $100 \mathrm{~W}$ HDLs, respectively. Motion sensors are currently in use for the 100 W HDL lighting systems. The CFLs will be directly installed to replace the HDLs without any modification on the existing lighting control systems. The exchanged HDLs and ICLs will be stored and destroyed, corresponding counting and crushing certificate of the disposed lamps will be issued by a disposal company. The CFLs with a rated lifetime of 10000 hours, manufactured by Philips, have the equivalent or higher lumen to the replaced HDLs and the ICLs. An energy audit is conducted to gather all relevant information of this project to help with the project design. Detailed information of this project is listed in Table 1.

Table 1: Details of the lighting project

\begin{tabular}{llll}
\hline Technology & Wattage & Operating Schedule & Quantity \\
\hline $\mathrm{ICL} \rightarrow \mathrm{CFL}$ & $60 \mathrm{~W} \rightarrow 14 \mathrm{~W}$ & $8: 00-16: 00$ & 263519 \\
$\mathrm{HDL} \rightarrow \mathrm{CFL}$ & $100 \mathrm{~W} \rightarrow 20 \mathrm{~W}$ & Motion sensor control & 140777 \\
\hline
\end{tabular}

The project participants conduct a cost analysis for the discussion of investment barrier. The average price of CFLs used in this project is $\mathrm{R} 27$ per $\mathrm{CFL}^{3}$, other financial details associated with this project can be found in Table 2 .

\subsection{Lighting baseline methodology}

The AMS-II.C. [11] is adopted to develop the energy baseline for this project. The AMS-II.C. offers indicative simplified baseline and monitoring methodologies for the demand-side

\footnotetext{
${ }^{3}$ The annual average USD to Rand exchange rate in 2012 is $1 \mathrm{USD}=\mathrm{R}$
}

Table 2: Summary of the cost analysis

\begin{tabular}{lrl}
\hline Total number of CFLs to be installed & 404296 & Units \\
\hline Costs & & \\
\hline Average price of CFL & 27 & Rand per unit \\
CFL distribution & 5 & Rand per unit \\
Subtotal & 12.94 & Million Rand \\
HDL, ICL, CFL collection, & & \\
transportation, and disposal & 1.03 & Million Rand \\
$\begin{array}{l}\text { CDM process cost } \\
\text { (PDD writing, validation, }\end{array}$ & & \\
monitoring and verification, etc) & & \\
\hline Total project cost & 1.72 & Million Rand \\
\hline
\end{tabular}

energy efficiency activities for specific technologies such as installing new energy efficiency lamps, ballasts, refrigerators, motors, fans, etc. There are several other approved CDM lighting methodologies that are available on the UNFCCC's website, such as: AM0046 [22], AMS-II.J. [23], AMS-II.N. [24], etc. The AM0046 is the first end-use energy efficiency methodology for distribution of CFLs to households. However, this guideline focuses on large scale CDM projects and the monitoring requirement of the project are very cumbersome [8]. The AMS-II.J. is actually a deemed savings methodology that has relaxed the heavy monitoring requirements of AM0046. However, the AMS-II.J. generates significantly less CERs than the other methodologies due to a very conservative assumption on average daily utilisation of CFLs. The AMS-II.N. is newly approved in March 2012 and it offers guidance to the demand-side energy efficiency projects for the installation of energy efficient lights and/or controls in building. Up to December 2012, this guideline has not been used in any registered CDM energy efficient lighting projects.

According to the AMS-II.C., if the lighting population is classified into $K$ groups, then the baseline energy for the lighting system is calculated by eq. (1)

$$
E_{B}=\sum_{i=1}^{K}\left(N_{i} \cdot P_{i} \cdot O_{i}\right)
$$

As discussed in the AMS-II.C., $P_{i}$ and $O_{i}$ may be determined separately or in combination, i.e., as energy consumption. In this study, $P_{i}$ and $O_{i}$ are considered in combination in order to simplify the uncertainty analysis of the measurement. Therefore, eq. (1) could be simplified into

$$
E_{B}=\sum_{i=1}^{K}\left(N_{i} \cdot E_{i}\right)=N \bar{E}
$$

where

$$
\bar{E}=\frac{\sum_{i=1}^{K}\left(N_{i} \cdot E_{i}\right)}{\sum_{i=1}^{K} N_{i}}
$$

When the energy consumption baseline $E_{B}$ multiplied by the number of days during the monitoring period and the relevant emission factor, the baseline emission of the involved lighting systems can be obtained. Energy consumption at the postimplementation stage can also be determined by eq. (2) when apply the monitored daily energy consumption of the CFLs. 


\subsection{Lighting classification and stratified random sampling}

The stratified random sampling approach is adopted for the sampling target since this sampling methodology is most applicable when there are obvious grouping of population elements whose characteristics are more similar within subgroups than across subgroups [19]. For the government hospital lighting project, the daily energy consumption patterns of the lighting population are not homogeneous. Therefore, the involved HDL and ICL lamps are naturally classified into two subgroups. Group I is the 263519 ICLs which have low uncertainties of daily energy consumption, and Group II is the 140777 HDLs which have high uncertainties of the daily energy consumption. The uncertainties in the two groups are characterised by $\mathrm{CV}$, which is defined as the standard deviation of the metering records divided by the mean. CV values are between 0 and 1 . If $\mathrm{CV}$ value is close to 0 , then it indicates that the uncertainty of measurement is small. Otherwise, if $\mathrm{CV}$ is close to 1 , then it means the monitored parameter of a sample group has large uncertainty. According to the sampling technology [25], a higher $\mathrm{CV}$ indicates more sample sizes are required to achieve reliable sampling results.

The estimated CV values for the aforementioned lighting project can be obtained as follows. During the on site energy audit, the daily utilisation hours for a small sample of the lamps with no control devices are recorded. The sampled lamps are burning 8 hours on average with a standard deviation of 1.5. The rated power of an ICL lamp is $60 \mathrm{~W}$ in Group I. Therefore, the estimated mean value of baseline daily energy consumption per lamp in Group I is $0.48 \mathrm{kWh}$ with the standard deviation of 0.09 . By the definition of $\mathrm{CV}$, the $\mathrm{CV}$ value of Group I is around 0.18 . To be conservative, $\mathrm{CV}$ of Group I is taken as 0.20. In Group II, the rated power of an HDL lamp is $100 \mathrm{~W}$. Although the lighting operating schedule is unknown, it could be assumed that on average the lamps in Group II are burning 6 hours per day with a low confidence by the energy audit. In this case, a CV value as high as 0.5 is historically recommended by [26] for the lamps in Group II. Thus, the estimated daily energy consumption of Group II is $0.6 \mathrm{kWh}$ with a standard deviation of 0.3 .

Since the energy consumption in Group II changes more frequently than that in Group I, the metering devices to be installed in Group II must have a very high sampling frequency based on Shannon' sampling theorem [27]. In addition, the meters to be installed in Group II need to have advanced control chips with high clock frequency and large memory capacity for the data storage. The metering device specifications of the two recommended meters $\left(M_{1}\right.$ and $\left.M_{2}\right)$ are provided in Table 3. The specifications indicate that the $M_{2}$ meters are capable of capturing the uncertainties in Group I. However, the $M_{1}$ meters are not applicable for the measurement of Group II.

Group I will be installed with less expensive meters to check its energy consumption variations, and Group II will be installed with expensive meters to monitor its real time energy consumption. According to [28], the key components of the metering cost include meter purchasing cost, installation cost and maintenance cost. The costs of $M_{1}$ and $M_{2}$ are listed in Table 4 as given by the meter company.
Table 3: Metering device specifications

\begin{tabular}{lll}
\hline Categories & $M_{1}$ & $M_{2}$ \\
\hline Voltage range (AC) & $150-270 \mathrm{~V}$ & $100-380 \mathrm{~V}$ \\
Current range & $50 \mathrm{~mA}-50 \mathrm{~A}$ & $10 \mathrm{~mA}-100 \mathrm{~A}$ \\
Accuracy & $\pm 0.01 \%$ & $\pm 0.002 \%$ \\
Time resolution & $300 \mathrm{~s}$ & $0.5 \mathrm{~s}$ \\
Memory capacity & $32 \mathrm{kB}$ & $8 \mathrm{MB}$ \\
\hline
\end{tabular}

Table 4: Metering equipment cost (per unit)

\begin{tabular}{lcc}
\hline Categories & Cost of $M_{1}$ & Cost of $M_{2}$ \\
\hline $\begin{array}{l}\text { Meter purchase } \\
\text { (once-off) }\end{array}$ & R 876 & R 3146 \\
$\begin{array}{l}\text { Meter installation } \\
\text { (once-off) }\end{array}$ & R 195 & R 320 \\
$\begin{array}{l}\text { Meter maintenance } \\
\text { (monthly) }\end{array}$ & R 45 & R 98 \\
\hline
\end{tabular}

The overall 90/10 criterion for this project can be maintained by letting Group I have a very high confidence/precision while letting Group II have a relatively low confidence/precision. This results in a greater number of less expensive meters being installed in Group I, and a smaller number of expensive meters being installed in group II in order to minimise the metering cost.

\subsection{Monitoring and sampling plan}

As mentioned in the introduction, more than 20 energy efficiency lighting projects have been registered under SSC CDM projects. For these projects, simple random sampling approach is adopted and sample sizes are generally decided by professional judgements or the experience from previous CDM projects. For instance, 200 meters are randomly installed to monitor the lighting population for the Visakhapatnam (India) OSRAM CFL distribution CDM project [20]. These registered CDM lighting projects do not seem to have considered the optimisation of the monitoring and sampling plan by taking advantage of lighting classification. In this study, an optimal monitoring and sampling plan is designed as follows.

1) The crediting period of this project is designed to be 10 years. The first monitoring report will be compiled 1 year after the implementation of this project, while other monitoring reports will be compiled in a fixed interval of every 3 years.

2) Meters will be randomly distributed and installed during baseline period to measure the daily energy consumption of each sampled lamp in both Group I and Group II for 3 calendar months. The sample sizes are decided by the proposed metering cost minimisation model. Each sampled lamp is monitored by one meter.

3) Once decided in the baseline period, the same sample sizes are applied at the post-implementation stage. The daily energy consumption of the sampled CFLs will be continuously measured during the crediting period.

4) Meters with different functionalities and prices will be applied in different lighting groups. The metering equipment 
will be installed by a meter company. Calibration and maintenance of the metering systems will be performed regularly.

\subsection{Uncertainty analysis and modelling assumptions}

According to the ASHRAE guideline 14 [29] and IPMVP 2012 [30], the uncertainties can be classified into 3 categories: the measurement uncertainty, modeling uncertainty and sampling uncertainty. Measurement uncertainties usually come from the inappropriate calibration of the measurement equipment, inexact measurement, or improper meter selection, installation and operation. The modelling uncertainties arises from the improper mathematical function form, inclusion of the irrelevant variables or exclusion of relevant variables. Sampling uncertainties are resulted from inappropriate sampling approaches or insufficient sample sizes.

In this study, only two parameters $N$ and $\bar{E}$ are involved in the lighting baseline model eq. (2). $N$ can be easily verified by the counting and crushing certificate. $\bar{E}$ will be obtained by the metering and sampling effort. As discussed in Subsection 2.3, the accuracy specifications of both the meters $M_{1}$ and $M_{2}$ are as high as $0.01 \%$ which are negligible when comparing to the required 90/10 criterion. Therefore, the sampling uncertainties will be the major concern in the metering cost minimisation modelling under the following assumptions.

1) Assume that no failure will happen to the metering system during the monitoring period since the meters are in good maintenance. In addition, the inflation of the maintenance cost will not be considered.

2) Let $X_{i}, i=1,2$ be the random variables that denote the baseline daily energy consumption data sets in Group I and Group II, respectively. From the well-known central limit theorem [31], it is assumed that $X_{1}$ and $X_{2}$ follow normal distributions, that is, $X_{i} \sim \mathcal{N}\left(\mu_{i}, \sigma_{i}^{2}\right)$. Then for any $n_{1}$ samples in Group I, the sampling distribution of the mean satisfies a normal distribution $\bar{X}_{1} \sim \mathcal{N}\left(\mu_{1}, \sigma_{1}^{2} / n_{1}\right)$ [32]. Similarly, the sampling distribution of the mean for any $n_{2}$ samples from Group II satisfies $\bar{X}_{2} \sim \mathcal{N}\left(\mu_{2}, \sigma_{2}^{2} / n_{2}\right)$.

3) Assume that $X_{1}$ and $X_{2}$ are independent. Then the sample mean for the overall project, which is defined as $\bar{E}$ previous will follow another normal distribution [32]

$$
\frac{N_{1} \bar{X}_{1}+N_{2} \bar{X}_{2}}{N} \sim \mathcal{N}\left(\frac{N_{1} \mu_{1}+N_{2} \mu_{2}}{N}, \frac{\sigma_{1}^{2}}{n_{1}} \cdot \frac{N_{1}^{2}}{N^{2}}+\frac{\sigma_{2}^{2}}{n_{2}} \cdot \frac{N_{2}^{2}}{N^{2}}\right)
$$

\subsection{Metering cost minimisation model}

As provided in [25], the initial sample size $n_{0}$ at the sampling plan stage can be calculated by

$$
n_{0}=\frac{z^{2} C V^{2}}{p^{2}}
$$

Fig. 1 plots the sample sizes against different $\mathrm{CV}$ values with some popular confidence/precision levels. Fig. 1 shows that

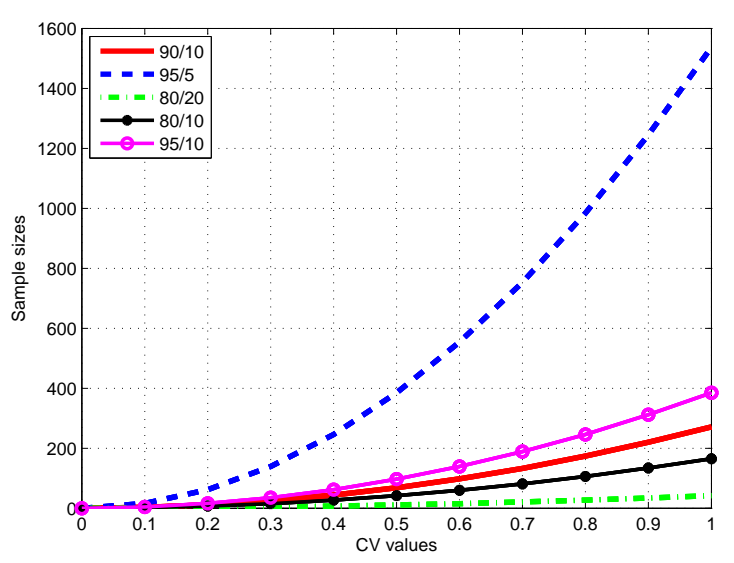

Figure 1: Sample sizes versus CV values

more sample sizes are required to achieve a higher confidence level and a better precision level for a given $\mathrm{CV}$ value.

The initial sample size $n_{0}$ that calculated by eq. (4) needs to be adjusted by the finite population correction factor [25]

$$
n=\frac{n_{0} N}{n_{0}+N}=\frac{C V^{2} z^{2} N}{C V^{2} z^{2}+N p^{2}} .
$$

For the $90 / 10$ criterion, $z=1.6450$ for $90 \%$ confidence and $p=10 \%$ as the allowed margin of error in eq. (5). The objective is to find the optimal solution of $\lambda=\left(z_{1}, z_{2}, p_{1}, p_{2}\right)$ which minimises

$$
f(\lambda)=\sum_{i=1}^{2}\left(a_{i}+b_{i}+k c_{i}\right) \times \operatorname{ceil}\left(n_{i}\right),
$$

where the ceil function $\operatorname{ceil}(\cdot)$ rounds a real number to the nearest integer which is greater than or equal to this real number. $\operatorname{ceil}\left(n_{i}\right)$ denotes the required sample sizes in the $i$-th group and $n_{i}$ can be calculated by eq. (5). For this project, $k=123$ including the baseline monitoring period ( 3 months) and the crediting period (10 years, 120 months).

Note that Group I is expected to have better confidence/precision than Group II, thus the two linear constraints $z_{1} \geq z_{2}$ and $p_{1} \leq p_{2}$ are obtained. The values of $z$ at various confidence levels are tabulated in many statistics books [33]. $z$ can be calculated by the $Z$-transformation formula

$$
z=\frac{\bar{x}-\mu}{\sigma / \sqrt{n}}
$$

and the constraints based on the 90/10 criterion are expressed 
as follows:

$$
\begin{aligned}
z & =\frac{\frac{N_{1} \bar{x}_{1}+N_{2} \bar{x}_{2}}{N}-\frac{N_{1} \mu_{1}+N_{2} \mu_{2}}{N}}{\sqrt{\left.\frac{\sigma_{1}^{2}}{n_{1}} \cdot\left(\frac{N_{1}}{N}\right)^{2}+\frac{\sigma_{2}^{2}}{n_{2}} \cdot\left(\frac{N_{2}}{N}\right)^{2}\right)}} \\
& =\frac{N_{1}\left(\bar{x}_{1}-\mu_{1}\right)+N_{2}\left(\bar{x}_{2}-\mu_{2}\right)}{\sqrt{\frac{\sigma_{1}^{2} N_{1}^{2}}{n_{1}}+\frac{\sigma_{2}^{2} N_{2}^{2}}{n_{2}}}} \\
& =\frac{N_{1} \frac{z_{1} \sigma_{1}}{\sqrt{n_{1}}}+N_{2} \frac{z_{2} \sigma_{2}}{\sqrt{n_{2}}}}{\sqrt{\frac{\sigma_{1}^{2} N_{1}^{2}}{n_{1}}+\frac{\sigma_{2}^{2} N_{2}^{2}}{n_{2}}}} \\
& \geq 1.6450,
\end{aligned}
$$

and

$$
\begin{aligned}
p & =\frac{N_{1} \bar{x}_{1}+N_{2} \bar{x}_{2}-\left(N_{1} \mu_{1}+N_{2} \mu_{2}\right)}{N_{1} \bar{x}_{1}+N_{2} \bar{x}_{2}} \\
& =\frac{N_{1} \frac{z_{1} \sigma_{1}}{\sqrt{n_{1}}+N_{2} \frac{z_{2} 2}{\bar{n}_{2}}}}{N_{1} \bar{x}_{1}+N_{2} \bar{x}_{2}} \\
& \leq 10 \% .
\end{aligned}
$$

In summary, the problem is to find $\lambda=\left(z_{1}, z_{2}, p_{1}, p_{2}\right)$ that minimises the objective function

$$
f(\lambda)=\sum_{i=1}^{2}\left(a_{i}+b_{i}+k c_{i}\right) \times \operatorname{ceil}\left(\frac{C V_{i}^{2} z_{i}^{2} N_{i}}{C V_{i}^{2} z_{i}^{2}+N_{i} p_{i}^{2}}\right),
$$

subject to the constraints

$$
\left\{\begin{array}{l}
z \geq 1.6450 \\
p \leq 10 \% \\
z_{1} \geq z_{2} \\
p_{1} \leq p_{2}
\end{array}\right.
$$

\section{Solution of the metering cost minimisation problem}

Before solving the optimisation problem, the metering cost to achieve the $90 / 10$ criterion without optimisation is calculated as a benchmark for comparison purposes. According to the energy audit and metering equipment cost in Table 4, the initial values to solve model eq. (10) are summarised in Table 5.

\begin{tabular}{lll}
\multicolumn{3}{c}{ Table 5: Initial values } \\
\hline Parameters & Group I & Group II \\
\hline Meter unit price & $a_{1}=\mathrm{R} 876$ & $a_{2}=\mathrm{R} 3146$ \\
Installation per meter & $b_{1}=\mathrm{R} 195$ & $b_{2}=\mathrm{R} 320$ \\
Monthly maintenance & $c_{1}=\mathrm{R} \mathrm{45}$ & $c_{2}=\mathrm{R} 98$ \\
Monitored months & $\mathrm{k}=123$ & $\mathrm{k}=123$ \\
CV values & $C V_{1}=0.20$ & $C V_{2}=0.50$ \\
Estimated $\bar{x}_{i}$ & $\bar{x}_{1}=0.48 \mathrm{kWh}$ & $\bar{x}_{2}=0.60 \mathrm{kWh}$ \\
Population sizes & $N_{1}=263519$ & $N_{2}=140777$ \\
\hline
\end{tabular}

For the government hospital lighting project, if the simple random sampling approach is applied to the entire lighting population, a worst possible CV value of 0.5 can be used for the sample size calculation by eq. (5), the obtained sample size is 68 with a metering cost of R 1055360 given that the expensive meters should be used when $\mathrm{CV}$ is high. For this solution, the 90/10 criterion is achieved without spending unnecessary money on metering. In this scenario, the metering cost shares $6.76 \%$ of the total project cost. However, without considering optimisation, another possible solution might be that the $90 / 10$ criterion is applied to Group I and II, where $\lambda=$ $(1.6450,1.6450,0.1,0.1)$. The corresponding sample sizes and metering cost are calculated as shown in Table 6. It shows that the overall confidence and precision are $97.76 \%$ and $9.94 \%$, respectively, at the total metering cost of R 1128 206, which occupies $7.19 \%$ of the total project cost. In this scenario, the expected sampling accuracy is higher than the required 90/10 criterion which is not necessary.

Table 6: Sample size and metering cost without optimisation

\begin{tabular}{llll}
\hline Parameters & Group I & Group II & Overall \\
\hline Confidence & $90 \%$ & $90 \%$ & $97.76 \%$ \\
Precision & $10 \%$ & $10 \%$ & $9.94 \%$ \\
Meter numbers & 11 & 68 & 79 \\
Number of samples & 11 & 68 & 79 \\
Total cost & R 72666 & R 1 055360 & R 1 128 026 \\
\hline
\end{tabular}

Now consider the metering cost minimisation model given in eq. (10) which is a nonlinear programming problem. For simplicity, the MATLAB function "fmincon" is applied to find the optimal solution of eq. (10). The optimisation settings of the "fmincon" function are shown as follows: the interior-point algorithm is chosen as the optimisation algorithm; the termination tolerance on the function value, 'TolFun', the termination tolerance on the constraint violation, 'TolCon', as well as the termination tolerance on the design variables, 'TolX' are all set to $10^{-15}$; in addition, "fmincon" also calculates the Hessian by a limited-memory, large-scale quasi-Newton approximation, where 20 past iterations are remembered. From a theoretical perspective, the sample size should be integer for the solution. Since this study focuses on practical issues of minimising the metering cost, real-valued sample sizes are used during the optimisation. After the optimal solution $\lambda=\left(z_{1}, z_{2}, p_{1}, p_{2}\right)$ is found, the ceil function is applied to calculate the integer sample size.

Besides these settings, a search starting point $\lambda_{0}$ as well as the boundaries of the design variable are also required for "fmincon" to work. Table 7 gives the optimal solution with $\lambda_{0}=(0.21,0.86,0.85,0.26)$, lower bound $l b=(0,0,0,0)$ and upper bound $u b=(+\infty,+\infty, 1,1)$.

\begin{tabular}{llll}
\multicolumn{4}{c}{ Table 7: Optimal results 1 } \\
\hline Parameters & Group I & Group II & Overall \\
\hline Confidence & $93.09 \%$ & $57.84 \%$ & $90.25 \%$ \\
Precision & $9.77 \%$ & $10.35 \%$ & $9.85 \%$ \\
Meter numbers & 14 & 16 & 30 \\
Number of samples & 14 & 16 & 30 \\
Total cost & R 92 484 & R 248 320 & R 340 804 \\
\hline
\end{tabular}

It is found that with the constraints of the 90/10 criterion for the overall project, the obtained confidence/precision 93.09/9.77 for Group I and 57.84/10.35 for Group II contribute to the overall $90.25 / 9.85$ accuracy. With these optimal $\left(z_{1}, z_{2}\right.$, $p_{1}, p_{2}$ ), the optimal metering cost is R 340804 , occupies $2.17 \%$ of the total project cost, which is largely reduced than the overall metering cost without the optimisation as given in Table 6. Comparing with the results in Table 6 , the metering cost for Group I increases, however, the metering cost of Group II reduces sharply. The overall metering cost is reduced whilst the 
$90 / 10$ criterion is satisfied.

Table 8: Optimal results 2

\begin{tabular}{llll}
\hline Parameters & Group I & Group II & Overall \\
\hline Confidence & $84.87 \%$ & $70.30 \%$ & $90.15 \%$ \\
Precision & $7.71 \%$ & $13.43 \%$ & $9.82 \%$ \\
Meter numbers & 14 & 16 & 30 \\
Number of samples & 14 & 16 & 30 \\
Total cost & R 92 484 & R 248 320 & R 340 804 \\
\hline
\end{tabular}

Due to the nonlinear nature of the minimisation problem, the optimal solutions may not be unique although the minimal objective function value is unique. Table 8 gives another optimal solution with the initial search point $\lambda_{0}=(2.1,2.4,0.62,0.22)$. It is clear that although the confidence and precision of the two groups are different from the values in Table 7, the 90/10 criterion is still achieved and the optimal sample sizes and the metering cost remain unchanged.

According to the discussions and analysis in this section, the metering costs with or without the optimisation are summarised in Tabel 9. Note that the figures in the "Metering cost (\%)" column are calculation against the total project cost of 15.69 million Rand. Comparing to the metering plan without the optimiation, the metering cost savings of 0.71 million Rand, which is $4.52 \%$ of the total project cost, are achieved without sacrificing the overall sampling accuracy.

\section{Model analysis and discussion}

The metering cost minimisation model in Section 3 is built for a particular CDM lighting retrofit project. When analysing model eq. (10), it is found that three key components, $M_{i}, C V_{i}$ and $N_{i}$ will affect the overall metering cost for a given accuracy requirement. Actually, for different CDM energy efficiency lighting projects, the population sizes $N_{i}$, the initial $\mathrm{CV}$ values $C V_{i}$ and the individual metering system cost $M_{i}$ may vary. In order to further analyse the metering cost reduction for other similar lighting energy efficiency projects, it is worth investigating how these variations will influence the optimal metering cost.

In the following, three simulations are run to characterise the impacts of the parameters $N_{i}, C V_{i}$ and $M_{i}$ to the optimal metering cost for similar lighting energy efficiency projects. For each simulation, only one parameter will change. More precisely, $N_{2}$ is changed in the first simulation to investigate the relationship between the population size and the optimal metering cost; $C V_{1}$ is changed in the second simulation to identify the relationship between initial $\mathrm{CV}$ values and the optimal metering cost; $M_{1}$ is changed in the third simulation to analyse the relationship between the individual metering system cost and the optimal metering cost. The optimal settings remain the same as the settings provided in Section 3. The search starting point is $\lambda_{0}=(0.21,0.86,0.85,0.26)$ for the three simulations. As mentioned in Section 3, due to the nonlinear nature of the minimisation problem, the optimal confidence/precision levels are not unique since there exist different combinations of optimal confidence/precision levels that satisfy the 90/10 criterion constraints. Different valid combinations of optimal confidence/precision levels are obtained by applying a different search starting point $\lambda_{0}$. Some artificial initial values are applied for the three simulations.

Results of the simulations are shown in Fig. 2 to Fig. 13. For the legends in Fig. 2, 3, 6, 7, 10, and 11, the confidence/precision levels of Group I and Group II are denoted by the dotted line (in red) and the dashed line (in blue), respectively. The confidence/precision levels for the overall project that are calculated by the real-valued sample sizes, are denoted by the solid line (in green) while the overall project confidence/precision levels calculated by the integer-valued sample sizes are denoted by the dash-dotted line (in black).

\subsection{Optimal metering cost versus population sizes}

In this simulation, let $N_{2}$ increase from $10 \quad 000$ to $N_{1}=1000000$ by a step of 10000 . Initial values for this simulation are listed in Table 10. The corresponding optimal results are presented in Fig. 2 Fig. 5.

Table 10: Initial values for the Simulation 1

\begin{tabular}{lll}
\hline Parameters & Group I & Group II \\
\hline$M_{i}$ & $M_{1}=\mathrm{R} 5000$ & $M_{2}=\mathrm{R} 50000$ \\
$C V_{i}$ & $C V_{1}=0.2$ & $C V_{2}=0.5$ \\
$\bar{x}_{i}$ & $\bar{x}_{1}=0.56 \mathrm{kWh}$ & $\bar{x}_{2}=0.36 \mathrm{kWh}$ \\
\hline
\end{tabular}

In Fig. 2, the confidence levels of Group I are always higher than those of Group II. The green solid line shows that the desired $90 \%$ confidence of the overall project is satisfied. However, since the sample size calculated by eq. (5) may not be integers, the ceil function is applied to round the real values of $n_{1}$ and $n_{2}$ to integers. The rounded $n_{1}$ and $n_{2}$ are used to calculate the overall project confidence and metering cost. It can be observed that the confidence levels calculated by the rounded $n_{1}$ and $n_{2}$ are greater than or equal to the project confidence calculated by the real-valued $n_{1}$ and $n_{2}$.

In Fig. 3, as $N_{2}$ increases, the precision levels of both Group I and Group II increase. The precision levels of Group II increase

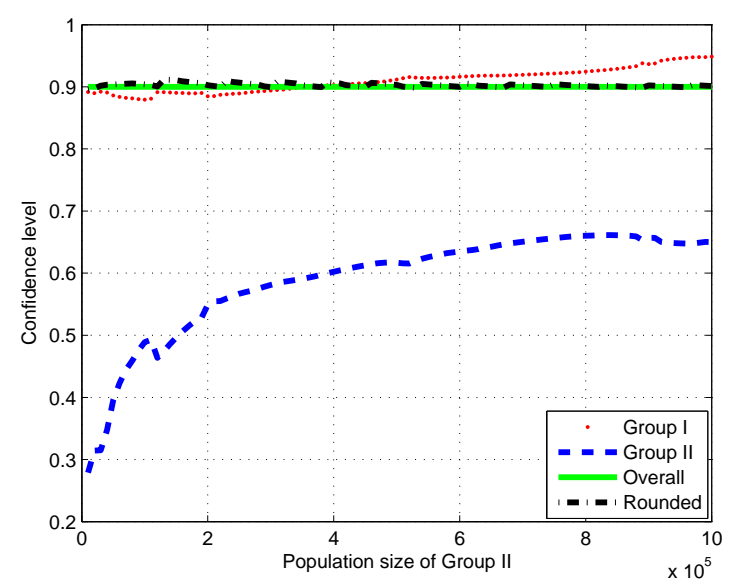

Figure 2: Confidence levels when $N_{2}$ changes 
Table 9: Metering cost analysis

\begin{tabular}{lrrrr}
\hline Solutions & Accuracy & Samples & Metering cost (R) & Metering cost (\%) \\
\hline No optimisation 1 & $90 / 10$ & 68 & R 1 055 360 & $6.76 \%$ \\
No optimisation 2 & $97.76 / 9.94$ & 79 & R 1 128 026 & $7.19 \%$ \\
Optimal solution 1 & $90.25 / 9.85$ & 30 & R 340 804 & $2.17 \%$ \\
Optimal solution 2 & $90.15 / 9.82$ & 30 & R 340 804 & $2.17 \%$ \\
\hline
\end{tabular}

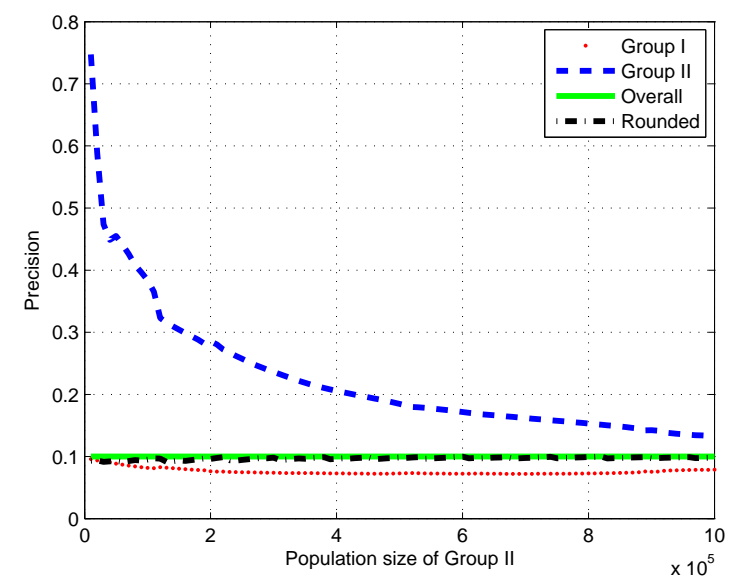

Figure 3: Precision levels when $N_{2}$ changes

more quickly than those of Group I in order to balance the overall project precision within $10 \%$. In addition, the project precision levels that calculated by the real-valued sample sizes and the integer-valued sample sizes are within the desired $10 \%$ precision.

In Fig. 4 and Fig. 5, it is clear that both the sample sizes and the metering cost increase as $N_{2}$ increases.

According to the results shown in Fig. 2 to Fig. 5, the influence of Group II to the overall accuracy is small when $N_{2}$ is small. When $N_{2}<10000$, only one meter is needed. The confidence for Group II is less than 50\% and the precision is poorer

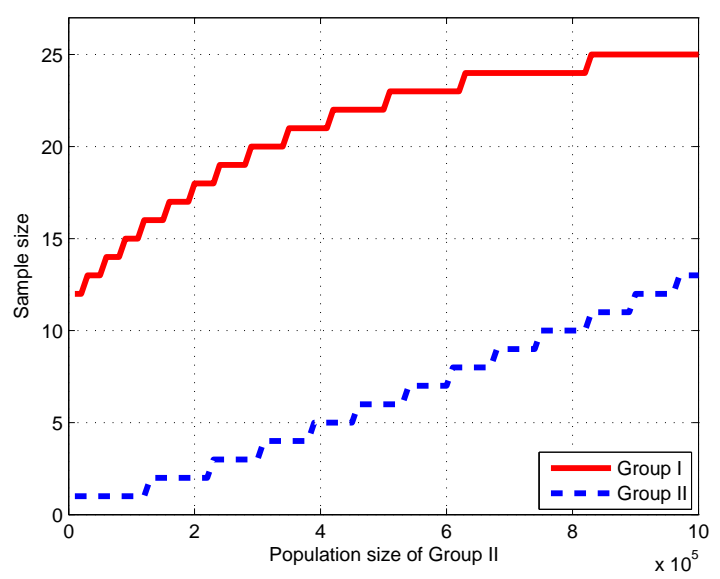

Figure 4: Number of meters when $N_{2}$ changes than $40 \%$ while the overall $90 / 10$ criterion can still be maintained. However, as $N_{2}$ increases, the uncertainties of Group II increase rapidly because $C V_{2}$ is high. Therefore, the required sample size of Group II goes up quickly.

\subsection{Optimal metering cost versus $C V$ values}

In this simulation, let $C V_{1}$ increase from 0.005 to $C V_{2}=0.5$ by a step of 0.005 . Initial values for this simulation are listed in Table 11. The optimal solutions are presented in Fig. 6 Fig. 9.

\begin{tabular}{lll}
\multicolumn{3}{c}{ Table 11: Initial values for the Simulation 2 } \\
\hline Parameters & Group I & Group II \\
\hline$M_{i}$ & $M_{1}=\mathrm{R} 5000$ & $M_{2}=\mathrm{R} 50000$ \\
$\bar{x}_{i}$ & $\bar{x}_{1}=0.56 \mathrm{kWh}$ & $\bar{x}_{2}=0.36 \mathrm{kWh}$ \\
$N_{i}$ & $N_{1}=1000000$ & $N_{2}=300000$ \\
\hline
\end{tabular}

Fig. 6 shows that the confidence levels of both Group I and Group II decrease when $C V_{1}$ increases. The confidence levels of Group I are higher than Group II. The overall confidence as shown in the solid line (in green) satisfies the $90 \%$ confidence. However, it is noted that the rounded overall confidence level is a little lower than the $90 \%$ confidence when $C V_{1}$ is between 0 and 0.1 . The reason is that real-valued sample sizes are allowed during the optimisation in this study. The optimal solutions for real-valued sample sizes may sometimes become suboptimal when ceil function is applied to these optimal solutions. In the worst case, the real-valued sample sizes satisfy the $90 \%$ confidence while the integer-valued sample sizes obtained by the ceil function do not satisfy the $90 \%$ confidence as shown in Fig. 6. In this case, it is suggested to increase necessary sample size

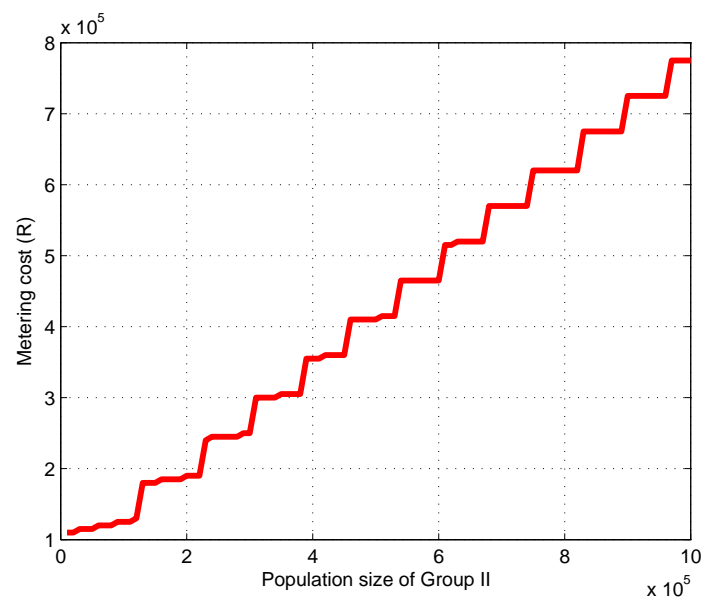

Figure 5: Metering cost when $N_{2}$ changes 


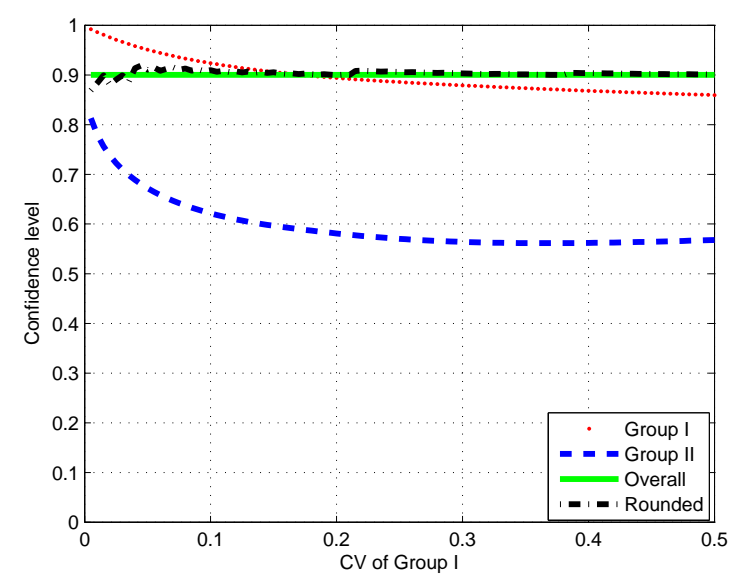

Figure 6: Confidence levels when $C V_{1}$ changes

to achieve the desired accuracy. To illustrate, consider the lowest confidence level $86.87 \%$ of the rounded overall confidence as shown by the first point in the dash-dotted line (in black) in Fig. 6. Detailed information for this point is listed in Table 12. From Table 12 it is clear that the confidence level in Group I is

\begin{tabular}{llll}
\multicolumn{4}{c}{ Table 12: Solution analysis } \\
\hline Parameters & Group I & Group II & Overall \\
\hline Confidence & $99.19 \%$ & $81.29 \%$ & $86.88 \%$ \\
Precision & $2.44 \%$ & $49.20 \%$ & $8.65 \%$ \\
Meter numbers & 1 & 2 & 3 \\
Total cost & R 5 000 & R 100 000 & R 105 000 \\
\hline
\end{tabular}

very close to $100 \%$. Calculation shows that even the confidence level in Group I increases to $99.99 \%$, the overall confidence can only achieve $89.36 \%$. Therefore, the only solution is to increase meters in Group II. When 3 meters are installed in Group II, the rounded overall confidence becomes $90.99 \%$ which meets the $90 \%$ confidence requirement. Since the installation of only 2 meters in Group II will never meet the 90/10 criterion, while the installation of 3 meters in Group II will meet the 90/10 criterion, this solution must be optimal.

In Fig. 7, it is noted that as $C V_{1}$ increases, the precision of Group I becomes worse but remains within $10 \%$. The precision of Group II improves but the precision is always worse than that of Group I. The overall precision maintains within the $10 \%$ margin of error.

In Fig. 8, the sample size of Group I keeps increasing as $C V_{1}$ goes up. It means that when $\mathrm{CV}$ increases, more samples are needed to maintain the $90 / 10$ criterion. The sample size of Group II also increases, but not as quick as Group I.

Fig. 9 shows the overall metering cost keeps going up since the sample sizes of both Group I and Group II increase.

The results provided in Fig. 6 to Fig. 9 show that, when $C V_{1}<0.1$, less than 10 samples can maintain the confidence of Group I over $90 \%$ and the precision within $10 \%$. However, as $C V_{1}$ becomes greater, the uncertainties of this group increase rapidly since the population size of Group I is dominant. There-

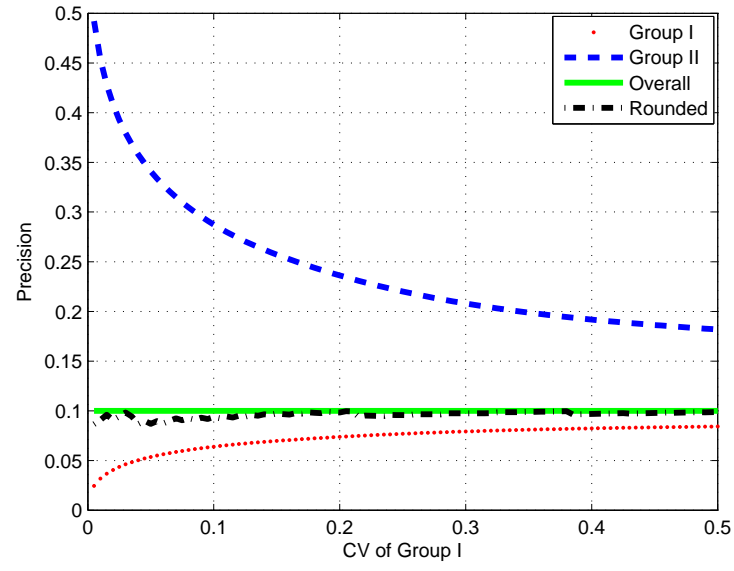

Figure 7: Precision levels when $C V_{1}$ changes

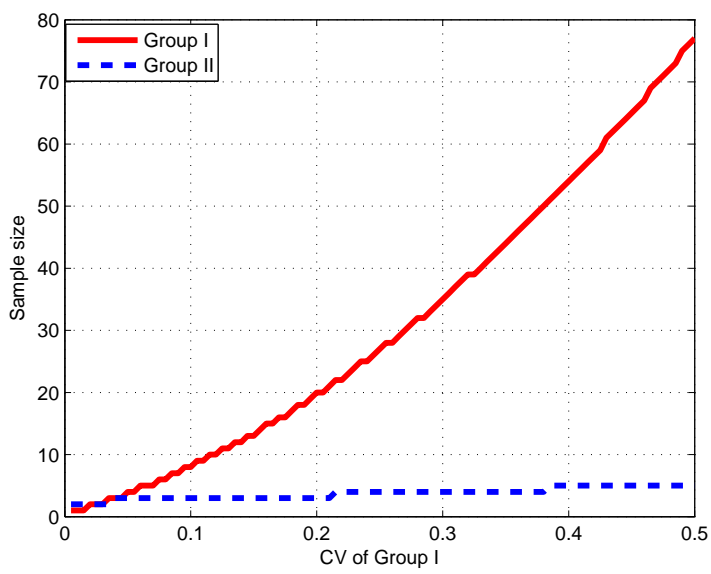

Figure 8: Number of meters when $C V_{1}$ changes

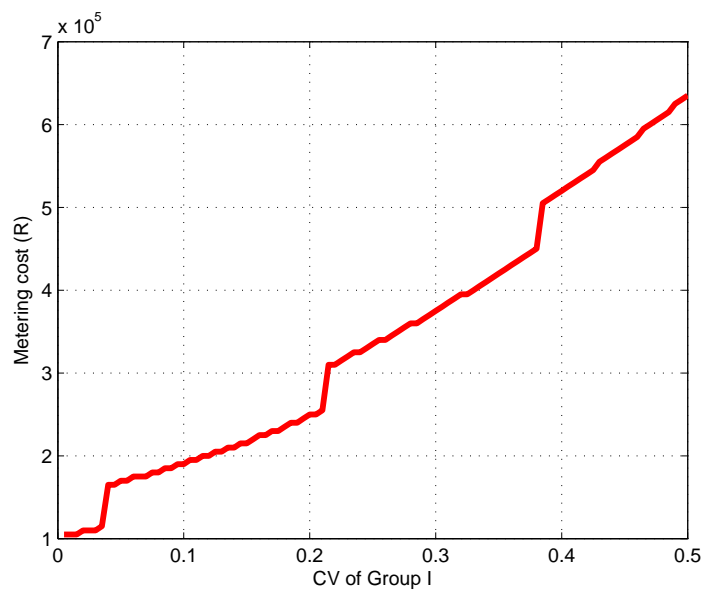

Figure 9: Metering cost when $C V_{1}$ changes 
fore the sample size of Group I increases more quickly.

\subsection{Optimal metering cost versus individual meter cost}

In this simulation, assume that the individual meter cost $M_{1}$ increases from 500 to $M_{2}=50000$ by a step of 500 . The initial values for this simulation are listed in Table 13. The optimal solutions are presented in Fig. 10 Fig. 13.

Table 13: Initial values for the Simulation 3

\begin{tabular}{lll}
\hline Parameters & Group I & Group II \\
\hline$C V_{i}$ & $C V_{1}=0.2$ & $C V_{2}=0.5$ \\
$\bar{x}_{i}$ & $\bar{x}_{1}=0.56 \mathrm{kWh}$ & $\bar{x}_{2}=0.36 \mathrm{kWh}$ \\
$N_{i}$ & $N_{1}=1000000$ & $N_{2}=300000$ \\
\hline
\end{tabular}

In Fig. 10, as $M_{1}$ increases, the confidence levels of both Group I and Group II go down slowly. The confidence levels of Group I are higher than those of Group II. Both the project confidence levels that calculated by the real-valued sample sizes and the integer-valued sample sizes satisfy the desired $90 \%$ confidence.

In Fig. 11, it is clear that the precision levels of Group II increase continually as $M_{1}$ goes up. However, the precision of Group I becomes worse since less meters are installed in Group I when $M_{1}$ increases.

In Fig. 12, the sample size of Group I decreases but the sample size of Group II increases as $M_{1}$ goes up.

Fig. 13 clearly shows that the general trend of the metering cost is going up when $M_{1}$ increases. Sometimes, the metering cost decreases a little because the sample size of Group I decreases while the sample size of Group II does not change.

Based on the results shown in Fig. 10 to Fig. 13, when $M_{1}$ is small, more samples are drawn from Group I to achieve both high confidence and precision. However, as $M_{1}$ increases, the sample size of Group I tends to decrease in order to achieve the minimal metering cost of the project.

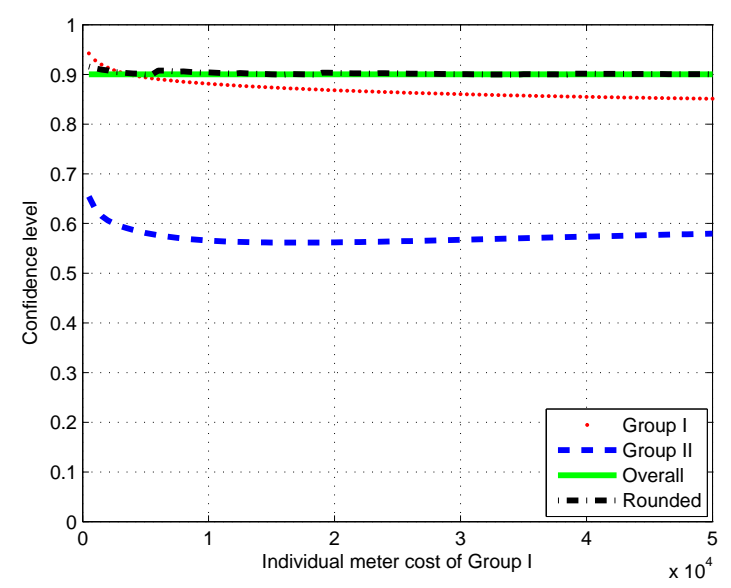

Figure 10: Confidence levels when $M_{1}$ changes

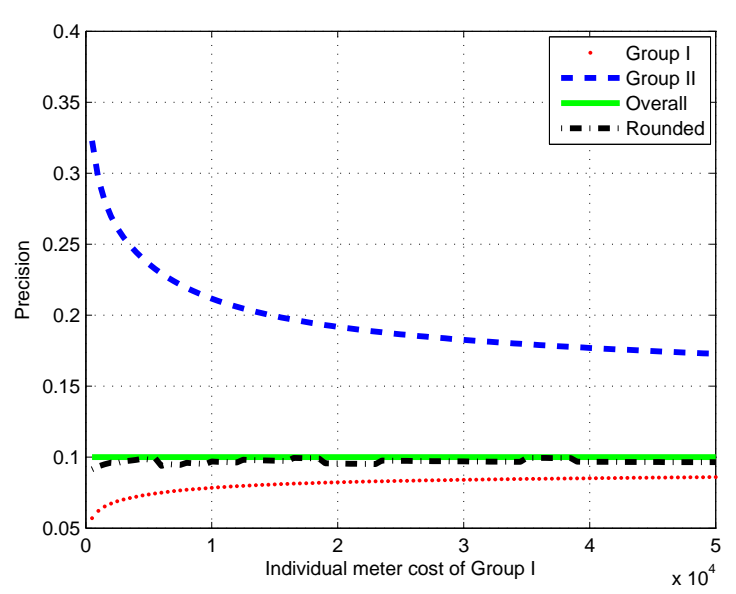

Figure 11: Precision levels when $M_{1}$ changes

\subsection{Remarks on the simulations}

The three simulations indicate that the proposed metering cost minimisation model is also applicable to reduce the metering cost for other similar energy efficiency lighting projects.

The simulation results also reveal the possibilities of further reducing the metering cost. For instance, instead of using the worst case of 0.5 as the $\mathrm{CV}$ value, the project participants may be willing to install a few less expensive meters to monitor the lighting daily energy consumption for a short period. In that scenario, it is more likely to obtain an estimated lighting daily energy consumption with a smaller $\mathrm{CV}$ value. A small $\mathrm{CV}$ means that less sample sizes are needed to achieve the accuracy requirements.

In addition, the simulation results can also be taken as an example of simplifying the metering cost minimisation methodology. In order to further simplify this methodology, it is suggested to pre-calculate and tabulate optimal metering cost and samples for similar energy efficiency lighting projects with dif-

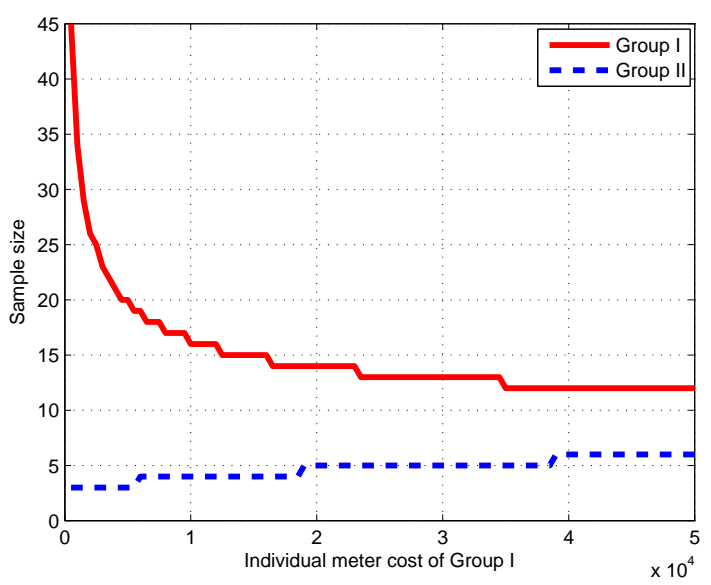

Figure 12: Number of meters when $M_{1}$ changes 


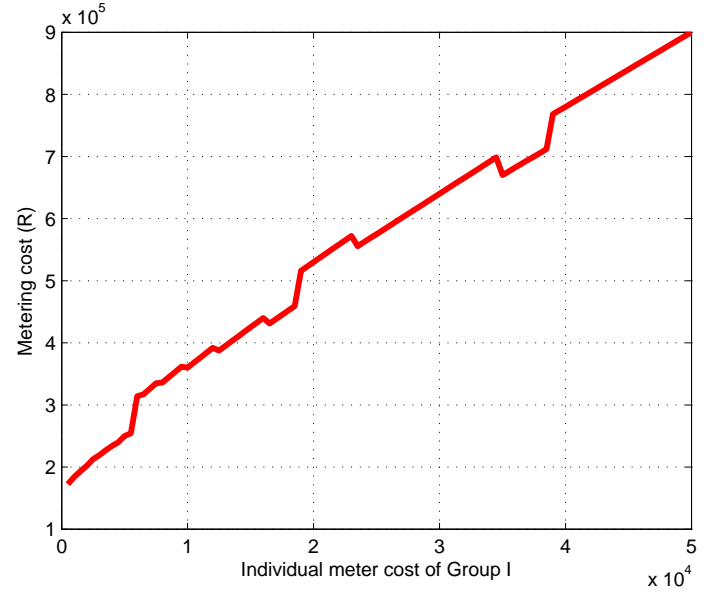

Figure 13: Metering cost when $M_{1}$ changes

ferent population sizes, $\mathrm{CV}$ values or metering device costs.

\section{Conclusion}

In this study, a metering cost minimisation model is proposed to assist the sampling plan of the CDM energy efficiency lighting project. The minimal metering cost is achieved by optimising the confidence and precision of each lighting group under the constraint of the 90/10 criterion for the overall project. In order to further analyse the metering cost reductions for other similar lighting energy efficiency projects, three simulations are conducted to investigate the relationships between the optimal metering cost and the population sizes of the groups, CV values and the individual meter equipment cost. The simulation results indicate that the proposed metering cost minimisation model can be applied to different CDM projects with different characteristics. In addition, the proposed model is also applicable to minimise the metering cost for project with more than two lighting groups. Moreover, this model can also be applied to projects with accuracy requirements other than the 90/10 criterion.

\section{Acknowledgement}

This work is supported by the National Hub for the Postgraduate Programme in Energy Efficiency and Demand Side Management at the University of Pretoria.

\section{References}

[1] Lee SJ, Ryu IS, Kim BM, Moon SH. A review of the current application of $\mathrm{N}_{2} \mathrm{O}$ emission reduction in CDM projects. International Journal of Greenhouse Gas Control 2011;5:167-76.

[2] UNFCCC. CDM Methodology Booklet. 2010. Available at: https://cdm.unfecc.int/methodologies.

[3] Mills E. Why we're here: the \$230-billion global lighting energy bill. Nice, France: Proceedings of the Right Light 5; 2002,.

[4] Wen YJ. Wireless sensor and actuator networks for lighting energy efficiency and user satisfaction. $\mathrm{PhD}$ thesis; Department of Mechanical Engineering, University of California; Berkeley, California; Fall 2008.
[5] Aydinalp M, Ugursal VI, Fung AS. Modeling of the appliance, lighting, and space-cooling energy consumptions in the residentail sector using neural networks. Applied Energy 2002;71:87-110.

[6] Jenkins D, Newborough M. An approach for estimating the carbon emissions associated with office lighting with a daylight contribution. Applied Energy 2007;84:608-22.

[7] Jaber JO. Future energy consumption and greenhouse gas emissions in Jordanian industries. Applied Energy 2002;71(1):15-30.

[8] Michaelowa A, Hayashi D, Marr M. Challenges for energy efficiency improvement under the CDM: the case of energy-efficient lighting. Energy Efficiency 2009;2:353-67.

[9] Mills E, Jacobson A. From carbon to light: a new framework for estimating greenhouse gas emissions reductions from replacing fuel-based lighting with LED systems. Energy Efficiency 2011;4:523-46.

[10] UNFCCC. General guidelines to SSC CDM methodologies. Technical Report; Version 19.0, 2012.

[11] UNFCCC. Approved small scale methodology AMS II.C.: demand-side energy efficiency activities for specific technologies. Technical Report; Version 14.0, 2012.

[12] UNFCCC. General guidelines for sampling and surveys for small-scale CDM project activities. Technical Report; Version 01, 2009.

[13] UNFCCC. Standard for sampling and surveys for CDM project activities and programme of activities. Technical Report; Version 03.0, 2012.

[14] Adcock CJ. Sample size determination: a review. The Statistician 1997;46(2):261-83.

[15] Nierwinski J. Reliability sampling methodology using simulation and re-sampling. IEEE Transactions on Reliability 2007;56:125-31.

[16] Liu W. On some sample size formulae for controlling both size and power in clinical trials. The Statistician 1997;46:239-51.

[17] Lindley DV. Theory and practice of bayesian statistics. Journal of the Royal Statistical Socity Series D 1983;32(1/2):1-11.

[18] Lindley DV. The choice of sample size. The Statistician 1997;46(2):129_ 38.

[19] UNFCCC. Guideline for sampling and surveys for CDM project activities and programme of activities. Technical Report; Version 02.0, 2012.

[20] UNFCCC. Project design document form: Visakhapatnam (India) OSRAM CFL distribution CDM project, Project 1754. Technical Report; Version 06, 2009. Available at: http://cdm.unfccc.int/Projects/DB/TUEVSUED1206629154.85/view.

[21] UNFCCC. Project design document form: Dubai CFL project, Project 6316. Technical Report; Version 03, 2012. Available at: http://cdm.unfccc.int/Projects/DB/AENOR1338463447.52/view.

[22] UNFCCC. Approved baseline and monitoring methodology AM0046: distribution of efficient light bulbs to household. Technical Report; Version 02,2007

[23] UNFCCC. Approved small scale methodology AMS II.J.: demand-side activities for efficient lighting technologies. Technical Report; Version 04, 2010.

[24] UNFCCC. Approved small scale methodology AMS II.N.: demand-side energy efficiency activities for installation of energy efficient lighting and or controls in buildings. Technical Report; Version 01.0, 2012.

[25] Thompson SK. Sampling. New York: John Wiley \& Sons, Inc.; second ed.; 2002.

[26] Department of Energy (USA). M\&V guidelines: measurement and verification for federal energy projects, Version 3.0. Technical Report; 2008. Available at: http://www.eere.energy.gov/femp.

[27] Shannon CE. Communication in the presence of noise. Proceedings of the Institute of Radio Engineers 1949;37(1):10-21.

[28] Department of Energy (USA). Metering best practice: a guide to achieving utility resource efficiency, Version 2.0. Technical Report; 2011.

[29] American Society of Heating Refrigerating and Air-conditioning Engineers (ASHRAE). ASHRAE guideline: measurement of energy and demand savings. Technical Report; 2002.

[30] Efficiency Valuation Organization (EVO). International performance measurement and verification protocol: concepts and options for determining energy and water savings, Volume 1. Technical Report; 2012.

[31] Fischer H. A history of the central limit theorem: from classical to modern probability theory. New York: Springer; first ed.; 2011.

[32] Witte RS, Witte JS. Statistics. Harcourt Brace College; 1997.

[33] Cochran WG. Sampling Techniques. New York: John Wiley \& Sons, Inc.; third ed.; 1997. 